\title{
Maternal Exposure to the Cannabinoid Agonist WIN $55,12,2$ during Lactation Induces Lasting Behavioral and Synaptic Alterations in the Rat Adult Offspring of Both Sexes
}

\author{
Andrew F. Scheyer, ${ }^{1,2,3}$ Milene Borsoi, ${ }^{1,2,3}$ Anne-Laure Pelissier-Alicot, ${ }^{1,2,3,4,5}$ and \\ (D) Olivier J.J. Manzoni ${ }^{1,2,3}$
}

https://doi.org/10.1523/ENEURO.0144-20.2020

${ }^{1}$ Institut de Neurobiologie de la Méditerranée, Institut National de la Santé et de la Recherche Médicale Unité 1249, Marseille, France, ${ }^{2}$ Aix-Marseille University, France, $13273,{ }^{3}$ Cannalab Cannabinoids Neuroscience Research International Associated Laboratory, Institut National de la Santé et de la Recherche Médicale-Aix-Marseille University/Indiana University, $13273,{ }^{4}$ Service de Psychiatrie, Assistance Public Hopitaux de Marseille, Centre Hospitalier Universitaire Conception, Marseille 13005, France, and ${ }^{5}$ Service de Médecine Légale, APHM, CHU Timone Adultes, Marseille 13005, France

\begin{abstract}
Consumption of cannabis during pregnancy and the lactation period is a rising public health concern (Scheyer et al., 2019). Exposure to synthetic or plant-derived cannabinoids via lactation disrupts the development of GABAergic neurons in the prefrontal cortex (PFC) and alters early-life behaviors (Scheyer et al., 2020b). Recently, additional data revealed that $\triangle 9$-tetrahydrocannabinol (THC) perinatal exposure via lactation causes lasting behavioral and neuronal consequences (Scheyer et al., 2020a). Here, the long-term effects in adult offspring of maternal exposure to the synthetic cannabinoid agonist WIN 55,12,2 are reported. The data demonstrate that rats exposed during lactation to WIN display social and motivational deficits at adulthood. These behavioral changes were paralleled by a specific loss of endocannabinoid-mediated long-term depression (eCB-LTD) in the PFC and nucleus accumbens (NAc), while other forms of synaptic plasticity remained intact. Thus, similarly to THC, perinatal WIN exposure via lactation induces behavioral and synaptic abnormalities lasting into adulthood.
\end{abstract}

Key words: accumbens; cannabinoid; lactation; perinatal; prefrontal cortex

\section{Significance Statement}

Consumption of cannabis during pregnancy and the lactation period is a rising public health concern. Exposure to synthetic or plant-derived cannabinoids via lactation disrupts perinatal programming in the prefrontal cortex (PFC) and early-life behaviors. Here, we explored the long-term effects of maternal exposure to the synthetic cannabinoid agonist WIN 55,12,2 in the adult offspring. The results indicate that rats exposed during lactation to WIN display social and motivational deficits at adulthood. These behavioral changes were paralleled by a specific loss of endocannabinoid-mediated long-term depression (eCB-LTD) in the PFC and nucleus accumbens (NAc), while other forms of synaptic plasticity remained intact.

Received April 10, 2020; accepted July 27, 2020; First published August 31, 2020.

The authors declare no competing financial interests.
Author contributions: A.F.S., M.B., A.-L.P.-A., and O.J.J.M. designed research; A.F.S. and M.B. performed research; A.F.S. and M.B. analyzed data; A.F.S. and O.J.J.M. wrote the paper. 


\section{Introduction}

Cannabis consumption by pregnant women is progressively increasing (Hurd et al., 2019; Scheyer et al., 2019). The principle psychoactive component of cannabis, $\Delta 9$ tetrahydrocannabinol (THC), in addition to other cannabinoids, is actively transferred to the developing infant via breastfeeding (Hurd et al., 2019; Scheyer et al., 2019). During the perinatal period (i.e., during prenatal and early postnatal development), the developing brain is acutely sensitive to exogenous cannabinoids (Scheyer et al., 2019). Exposure to cannabinoids via lactation alters the developmental trajectory of the prefrontal cortex (PFC), which has been identified as a cortical hub essential to planning, cognitive flexibility, and emotional behaviors (Goldman-Rakic, 1991) and a common target in various endocannabinoid (eCB)-related synaptopathies (Araque et al., 2017). Thus, exposure of lactating females to either THC, or a synthetic agonist of CB1R, altered the maturational trajectory of GABAergic transmission and led to behavioral abnormalities in early life (Scheyer et al., 2020b). Disruptions to GABAergic development are known to occur following adolescent exposure to THC, as well (Renard et al., 2017). Furthermore, perinatal THC exposure via lactation elicits lasting, deleterious impacts on social behavior and synaptic plasticity in the PFC of the adult offspring (Scheyer et al., 2020a).

Here, we investigated the effects in both sexes of adult offspring of maternal exposure to the synthetic cannabinoid agonist, WIN 55,12,2 (WIN). These data demonstrate that rats exposed during lactation to WIN display social and motivational deficits at adulthood. These behavioral changes were paralleled by a specific loss of eCB-mediated long-term depression (eCB-LTD) in the PFC and nucleus accumbens (NAc), while other forms of synaptic plasticity remained intact. Thus, perinatal WIN exposure via lactation induces behavior and synaptic abnormalities lasting into adulthood.

\section{Materials and Methods}

\section{Animals}

Animals were treated in compliance with the European Communities Council Directive (86/609/EEC) and the United States National Institutes of Health Guide for the Care and Use of Laboratory Animals. All animal procedures were performed in accordance with the Aix-Marseille University and INSERM animal care committee's regulations. All rats were group-housed with 12/12 h light/dark

This work was supported by the Institut National de la Santé et de la Recherche Médicale (INSERM), the INSERM-National Institutes of Health (NIH) Exchange Program (A.F.S.), the Fondation pour la Recherche Médicale Grant Equipe FRM 2015 (to O.J.J.M.), and the NIH Grant R01DA043982 (to O.J.J.M.).

Acknowledgements: We thank the Chavis-Manzoni team members for helpful discussions.

Correspondence should be addressed to Olivier J. J. Manzoni at olivier. manzoni@inserm.fr.

https://doi.org/10.1523/ENEURO.0144-20.2020

Copyright (C) 2020 Scheyer et al.

This is an open-access article distributed under the terms of the Creative Commons Attribution 4.0 International license, which permits unrestricted use, distribution and reproduction in any medium provided that the original work is properly attributed. cycles with ad libitum access to food and water [zeitgeber time $(Z T) 0=7$ A.M.]. All behavioral, biochemical and synaptic plasticity experiments were performed on male and female RjHan:wi-Wistar rats (> post-natal day (P)90) from pregnant females obtained from Janvier Labs. Pregnant dams arrived at embryonic day (E15) and remained undisturbed until delivery. Newborn litters found before 5 P.M. were considered to be born that day (P0). Dams were injected daily subcutaneously from P01 to P10 with WIN $(0.5 \mathrm{mg} / \mathrm{kg} / \mathrm{d})$, dissolved in $10 \%$ polyethylene glycol $/ 10 \%$ Tween/80\% saline and injected subcutaneously (Borsoi et al., 2019). Control dams (sham) received vehicle.

\section{Behavioral procedures}

\section{Open field}

Observations were conducted after rats were adapted to the room laboratory conditions for at least $1 \mathrm{~h}$ before testing. Tests were conducted in a $45 \times 45 \mathrm{~cm}$ transparent Plexiglas arena. All behavioral procedures were performed between 10 A.M. and 3 P.M. A video tracking system (Ethovision XT, Noldus Information Technology) recorded the total distance traveled and time spent in the central zone $(21 \times 21 \mathrm{~cm})$ of the apparatus (Borsoi et al., 2019).

\section{Social interaction}

The apparatus consisted of a transparent acrylic chamber $(120 \times 80 \mathrm{~cm})$ divided into three equal compartments $(40 \mathrm{~cm}$ each) partially separated by white walls. The central compartment was empty and lateral compartments had an empty wire cage $(20 \mathrm{~cm}$ in diameter) were an object or a new rat (social stimulus) were placed during the test. WIN or sham-exposed rats were individually habituated to the test cage containing the two empty wire cages for $5 \mathrm{~min}$ immediately before testing. The first trial (social approach, 5-min duration) consisted of giving the tested rat the option to socialize with either a novel object or a new, naive, age-mate, and sex-mate conspecific rat that were placed into the wire cages positioned on the arena's opposite sides; $30 \mathrm{~min}$ later, the tested rat returned to the apparatus for the second trial (social memory, 5-min duration) wherein the two compartments held either the now-familiar rat from the first testing phase or a second, previously unknown, naive, agemate, and sex-mate conspecific.

Only rats with no compartment preference during the habituation phase were used. Time spent in each compartment and time spent exploring wire cages during the social approach and social memory phases were scored. Social preference ratio was calculated as time spent exploring either the wire cage containing the object, or the new rat divided by total time exploring both wire cages. Likewise, social memory ratio was calculated as time spent exploring either the wire cage containing the rat used in the first trial or the new rat divided by total time exploring both wire cages. Recognition index $>0.5$ indicates preferable object recognition memory.

\section{Anhedonia \\ We performed sucrose consumption tests (Monleon et al., 1995; Bessa et al., 2009). Rats were exposed for $24 \mathrm{~h}$}


to a bottle containing a sucrose solution ( $5 \%$ in tap water, Sigma), placed in the wire-top cage cover adjacent to standard tap water, followed by $12 \mathrm{~h}$ of water deprivation and a 20-min exposure to two identical bottles (one filled with $5 \%$ sucrose solution and the other with water). Bottles were placed at opposite ends of the cage and counterbalanced across groups to avoid side bias. Sucrose preference was calculated as the ratio of the volume of sucrose versus volume or consumed during the 20-min test. All animals were habituated to the testing room $24 \mathrm{~h}$ before initiating the sucrose preference test.

\section{Slice preparation}

Adult male and female rats were anesthetized with isoflurane and killed (Bara et al., 2018; Borsoi et al., 2019). The brain was sliced $(300 \mu \mathrm{m})$ in the coronal plane with a vibratome (Integraslice, Campden Instruments) in a sucrose-based solution at $4^{\circ} \mathrm{C}(87 \mathrm{~mm} \mathrm{NaCl}, 75 \mathrm{~mm}$ sucrose, $25 \mathrm{~mm}$ glucose, $2.5 \mathrm{~mm} \mathrm{KCl}, 4 \mathrm{~mm} \mathrm{MgCl}, 0.5 \mathrm{~mm} \mathrm{CaCl}_{2}$, $23 \mathrm{~mm} \mathrm{NaHCO}_{3}$, and $1.25 \mathrm{~mm} \mathrm{NaH} \mathrm{PO}_{4}$ ). Immediately after cutting, slices containing the medial PFC or the NAC were stored for $1 \mathrm{~h}$ at $32^{\circ} \mathrm{C}$ in a low-calcium artificial CSF (ACSF) that contained the following: $130 \mathrm{~mm} \mathrm{NaCl}, 11 \mathrm{~mm}$ glucose, $2.5 \mathrm{~mm} \mathrm{KCl}, 2.4 \mathrm{~mm} \mathrm{MgCl}_{2}, 1.2 \mathrm{~mm} \mathrm{CaCl}_{2}, 23 \mathrm{~mm}$ $\mathrm{NaHCO}_{3}$, and $1.2 \mathrm{~mm} \mathrm{NaH} \mathrm{PO}_{4}$, and were equilibrated with $95 \% \mathrm{O}_{2} / 5 \% \mathrm{CO}_{2}$ and then at room temperature until the time of recording. During the recording, slices were placed in the recording chamber and superfused at $2 \mathrm{ml} /$ min with low $\mathrm{Ca}^{2+}$ or normal $\mathrm{Ca}^{2+}$ ACSF (PFC and NAc, respectively). All experiments were done at $32^{\circ} \mathrm{C}(\mathrm{PFC})$ or $25^{\circ} \mathrm{C}$ (NAc). The superfusion medium contained picrotoxin (100 mm) to block GABA-A receptors. All drugs were added at the final concentration to the superfusion medium.

\section{Electrophysiology}

Whole-cell patch clamp of visualized layer five pyramidal medial PFC or medium spiny neurons (MSNs) and field potential recordings were made in coronal slices using standard procedures (Bara et al., 2018; Borsoi et al., 2019). Neurons were visualized using an upright microscope with infrared illumination. The intracellular solution was based on $\mathrm{K}^{+}$gluconate $\left(145 \mathrm{mM} \mathrm{K}^{+}\right.$gluconate, 3 $\mathrm{mm} \mathrm{NaCl}, 1 \mathrm{~mm} \mathrm{MgCl}$, 1 mм EGTA, $0.3 \mathrm{~mm} \mathrm{CaCl}$, 2 mM $\mathrm{Na}^{2+}$ ATP, $0.3 \mathrm{~mm} \mathrm{Na}^{+}$GTP, and $0.2 \mathrm{~mm}$ cAMP, buffered with 10 HEPES). The $\mathrm{pH}$ was adjusted to 7.2 and osmolarity to 290-300 mOsm. Electrode resistance was 4-6 M $\Omega$. Recordings were performed with an Axopatch-200B amplifier. Data were low pass filtered at $2 \mathrm{kHz}$, digitized (10 kHz, DigiData 1440A, Axon Instrument), collected using Clampex 10.2, and analyzed using Clampfit 10.2 (all from Molecular Device).

A $-2-m V$ hyperpolarizing pulse was applied before each evoked EPSC to evaluate the access resistance and those experiments in which this parameter changed $>25 \%$ were rejected. Access resistance compensation was not used, and acceptable access resistance was $<30 \mathrm{M} \Omega$. The potential reference of the amplifier was adjusted to zero before breaking into the cell. Cells were held at $-75 \mathrm{mV}$.
Current-voltage (I-V) curves were made by a series of hyperpolarizing to depolarizing current steps immediately after breaking into the cell. Membrane resistance was estimated from the I-V curve around resting membrane potential (Martin et al., 2015). Field potential recordings were made in coronal slices containing the PFC or the NAc (Kasanetz et al., 2013). During the recording, slices were placed in the recording chamber and superfused at $2 \mathrm{ml} /$ min with low $\mathrm{Ca}^{2+}$ ACSF. All experiments were done at $32^{\circ} \mathrm{C}$. The superfusion medium contained picrotoxin (100 $\mathrm{mm}$ ) to block GABA-A receptors. All drugs were added at the final concentration to the superfusion medium. The glutamatergic nature of the field EPSP (fEPSP) was systematically confirmed at the end of the experiments using the ionotropic glutamate receptor antagonist CNQX (20 $\mathrm{mm}$ ), which specifically blocked the synaptic component without altering the non-synaptic.

Both fEPSP area and amplitude were analyzed. Stimulation was performed with a glass electrode filled with ACSF and the stimulus intensity was adjusted $\sim 60 \%$ of maximal intensity after performing an input-output curve (baseline EPSC amplitudes ranged between 50 and $150 \mathrm{pA}$ ). Stimulation frequency was set at $0.1 \mathrm{~Hz}$.

\section{Data acquisition and analysis}

The magnitude of plasticity was calculated at $0-10$ and 30-40 min after induction (for Theta Burst Stimulation (TBS)LTP and eCB-LTD) or drug application (mGlu2/3-LTD) as percentage of baseline responses. Statistical analysis of data was performed with Prism (GraphPad Software) using tests indicated in the main text after outlier subtraction (Grubb's test, $\alpha$ level 0.05). All values are given as mean \pm SEM, and statistical significance was set at $p<0.05$.

\section{Results}

In rodent models, exposure to cannabinoids (both synthetic and plant-derived) during gestation or early development induces an array of deleterious consequences on behavior manifesting both at early life and adulthood (Hurd et al., 2019; Scheyer et al., 2019).

Perinatal exposure via lactation to either the plant-derived phytocannabinoid THC, or the synthetic cannabinoid, WIN, induces a significant delay in the trajectory of GABAergic development in the PFC of developing offspring, an effect which is accompanied by substantial behavioral alterations (Scheyer et al., 2020b). Further, the progeny of dams similarly exposed via lactation to THC during the first $10 \mathrm{~d}$ of postnatal life exhibit lasting deficits in synaptic plasticity in the PFC as well as augmented social behavior at adulthood (Scheyer et al., 2020a).

Here, we used this same protocol of perinatal cannabinoid exposure to determine whether synaptic and behavioral consequences are similarly produced following maternal administration of WIN. Thus, lactating dams were treated with a low dose of WIN $(0.5 \mathrm{mg} / \mathrm{kg}$, s.c.) or its vehicle (herein referred to as sham) from postnatal day (PND)1 to PND10. Experiments were then conducted in the male and female offspring at adulthood (>PND90).

All treatment effects (e.g., sham vs WIN) were found to be consistent across sexes. Thus, for figures and 
Table 1: Social approach and social memory data by sex

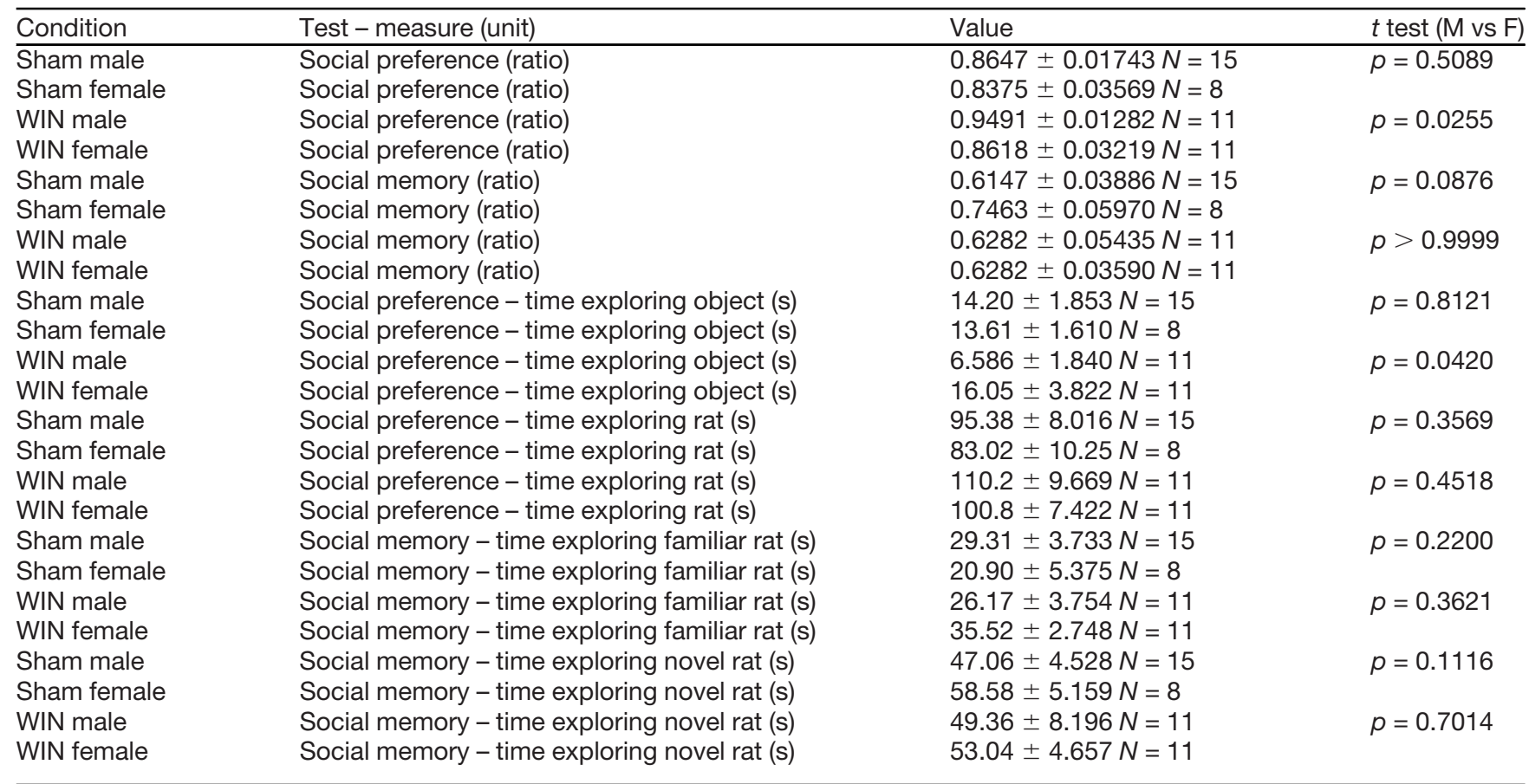

statistical analyses, data for male and female rats within treatment condition were combined. However, differences between the sexes within treatment conditions were noted in some measures. Details of within-treatment sex differences can be found in Tables 1-8.

\section{Perinatal exposure to a synthetic cannabimimetic alters social behavior and memory at adulthood}

In order to determine whether the behavioral repertoire of WIN-exposed animals is altered at adulthood, we performed several behavioral analyses in both male and female rats. Because perinatally $\mathrm{THC}$-exposed animals exhibited augmented social behavior at adulthood, we initiated a social approach and memory assay (Fig. 1A-D; Table 1).

First, during the social approach portion of the assay, WIN-exposed animals exhibited significantly heightened preference for a novel rat over a novel object, as compared with sham rats (Fig. 1A). Both sham-exposed and WIN-exposed rats exhibited a significant preference for the social stimulus as compared with the object (Fig. 1B). However, the magnitude of difference between time spent exploring the novel rat versus the novel object was significantly heightened in the adult offspring of WIN-treated dams. During the subsequent memory test, both shamexposed and WIN-exposed rats exhibited a similar social preference for a novel rat over the familiar rat from the social approach assay (Fig. 1C,D).

Further, naturalistic behavior was observed before the social approach/memory testing by observing animals in the open field assay. No significant differences were noted in the time spent in the left of the arena, distance covered during the trial, or the exploratory behavior (number of rearing events) during the open field test (Fig. 1E-G; Table 2).

\section{Perinatal exposure to WIN alters prefrontal synaptic plasticity at adulthood}

Perinatal THC exposure alters several forms of synaptic plasticity in the PFC at adulthood (Scheyer et al., 2020a). Thus, we elected to examine three forms of PFC plasticity to determine whether similar alterations followed perinatal WIN exposure. First, we used a $10 \mathrm{~min}, 10-\mathrm{Hz}$ stimulation of superficial layers of the PFC to elicit an eCB-LTD at deep layer synapses (Fig. 2A,B; Table 3). Here, we found that while sham-exposed rats exhibited robust, lasting depression 30-40 min following the 10-min protocol, no such LTD was observed in the PFC of WIN-exposed rats. This finding is in line with our previous data showing an ablation of eCB-LTD in the PFC of THC-exposed rats.

Next, we examined a distinct form of LTD in the PFC mediated by mGlu2/3 receptors (Bara et al., 2018) which has previously been shown to be disrupted by chronic exposure to drugs (Hoffman et al., 2003; Huang et al., 2007; Kasanetz et al., 2013) and augmented at adulthood following perinatal THC exposure (Scheyer et al., 2020a). Thus, we exposed acute PFC slices to the mGlu2/3 agonist LY379268 (300 nM) to elicit an mGlu2/3-dependent LTD (Fig. 2C,D; Table 3). Here, we found that PFC synapses in slices obtained from the offspring of both shamtreated and WIN-treated dams exhibited a similar magnitude of mGlu2/3-dependent LTD at 30-40 min following drug application.

Finally, we used a $\theta$-burst stimulation protocol at superficial layers of the PFC to induce a lasting synaptic potentiation (TBS-LTP) at deep layer synapses. Here, we found no alterations to the time course or magnitude of plasticity 
A

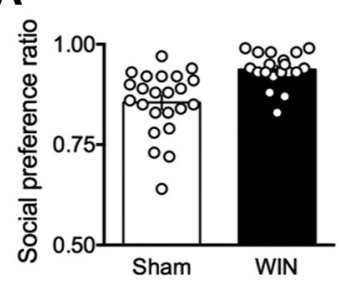

C

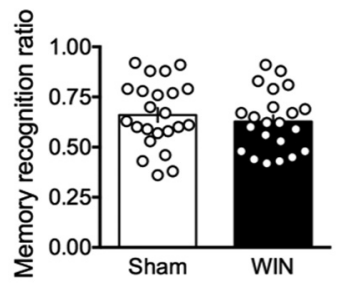

B

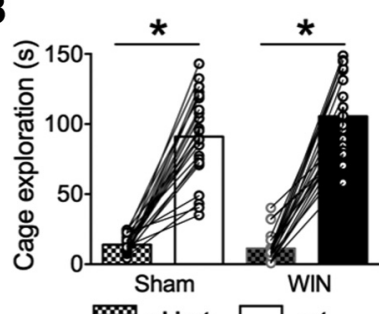

D

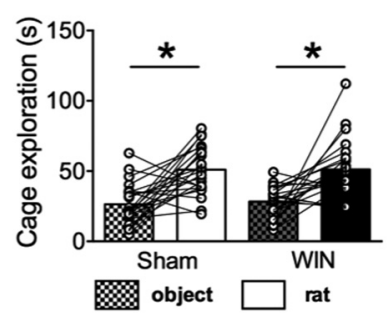

E

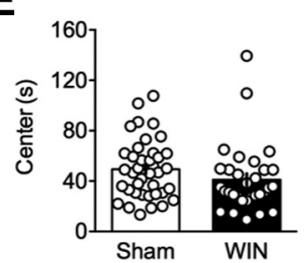

$\mathbf{F}$

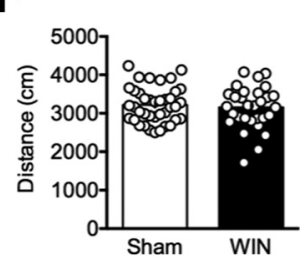

G

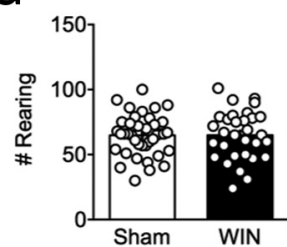

Figure 1. Perinatal WIN alters social approach, but not social memory behavior, nor behavior in the open field environment. $\boldsymbol{A}$, Adult offspring of WIN-treated dams exhibit significantly higher social preference than those of sham-treated dams (two-tailed $t$ test, $p=0.0001$; $N=23,19$, respectively). $\boldsymbol{B}$, Time spent exploring a novel rat is significantly higher than time spent exploring a novel object for both sham-exposed and WIN-exposed rats (one-way ANOVA, $F_{(3,86)}=14.49$; Tukey's post hoc analysis, $p<0.0001$ for both groups; $N=23$, 19 , respectively). $\boldsymbol{C}, \boldsymbol{D}$, In the subsequent social memory test, the adult offspring of both sham-treated and WIN-treated dams exhibited significantly higher preference for a novel, as compared with familiar, rats. $\boldsymbol{C}$, The social memory index does not differ between the two groups (two-tailed $t$ test, $p=0.557$ ). $\boldsymbol{D}$, Time spent exploring a novel rat is significantly higher than time spent exploring a familiar rat for both sham-exposed and WIN-exposed rats (one-way ANOVA, $F_{(3,86)}=2.137$; Tukey's post hoc analysis, $p<0.0001$ for both groups). $E$ G, Behavior in the open field environment does not differ between the offspring of sham-treated and WIN-treated dams $(N=39$, 31, respectively). Time spent in the left of the arena, total distance covered, and the number of rearing events is not significantly different between groups (two-tailed $t$ tests, $p=0.1817, p=0.5991$, and $p=0.9783$, respectively); $* p<0.05$.

between slices obtained from the adult offspring of shamtreated, as compared with WIN-treated, dams (Fig. 2E,F; Table 4). Of note, these results stand in contrast to those from THC-exposed rats, wherein TBS-LTP is impaired at adulthood (Scheyer et al., 2020a).

Because perinatal THC exposure altered parameters of cell excitability in the PFC at adulthood, we next sought to determine whether WIN exposure elicited similar augmentations in excitability. Interestingly, pyramidal neurons in PFC slices obtained from the adult offspring of shamtreated and WIN-treated dams did not differ with regards to input-output excitability, spikes elicited by progressive current injections, nor in the rheobase or resting membrane potential (Fig. 3A-D; Table 5).

Table 2: Open field data by sex

\begin{tabular}{|c|c|c|c|}
\hline Condition & Test - measure (unit) & Value & $t$ test (M vs F) \\
\hline Sham male & Open field - distance (cm) & $3120 \pm 80.10 N=22$ & $p=0.1490$ \\
\hline Sham female & Open field - distance $(\mathrm{cm})$ & $3348 \pm 135.8 N=17$ & \\
\hline WIN male & Open field - distance (cm) & $2861 \pm 151.9 N=14$ & $p=0.0070$ \\
\hline WIN female & Open field - distance $(\mathrm{cm})$ & $3396 \pm 97.26 N=17$ & \\
\hline Sham male & Open field - rearing (\#) & $62.23 \pm 3.365 N=22$ & $p=0.2443$ \\
\hline Sham female & Open field - rearing (\#) & $68.18 \pm 3.735 N=17$ & \\
\hline WIN male & Open field - rearing (\#) & $56.64 \pm 5.840 N=14$ & $p=0.0333$ \\
\hline WIN female & Open field - rearing (\#) & $71.76 \pm 3.118 N=17$ & \\
\hline Sham male & Open field - left (s) & $54.85 \pm 5.348 N=22$ & $p=0.0839$ \\
\hline Sham female & Open field - left (s) & $42.24 \pm 4.674 N=17$ & \\
\hline WIN male & Open field - left (s) & $55.87 \pm 8.835 N=14$ & $p=0.0104$ \\
\hline WIN female & Open field - left (s) & $28.92 \pm 2.733 N=17$ & \\
\hline
\end{tabular}


A

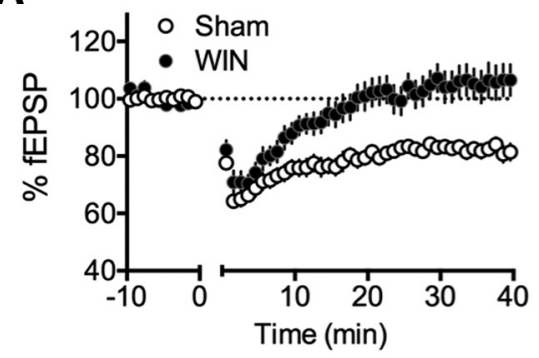

C

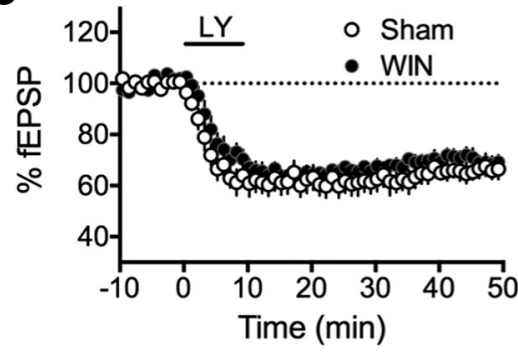

E

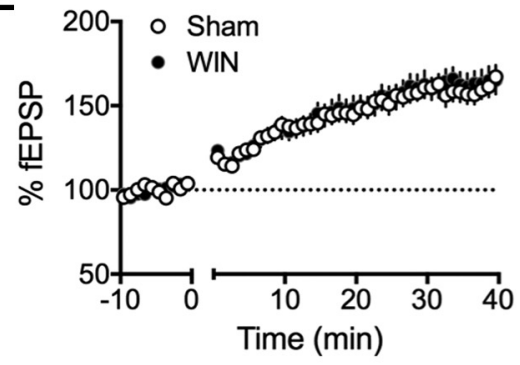

B

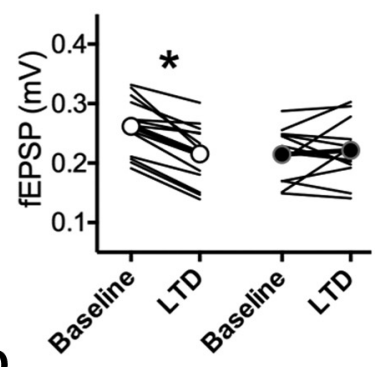

D

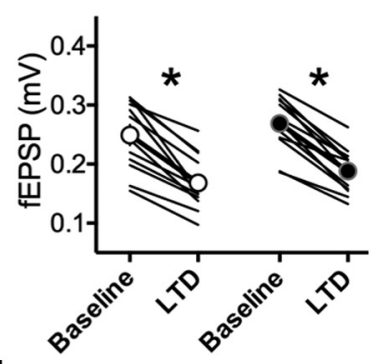

F

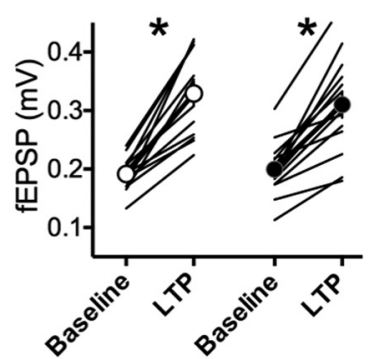

Figure 2. Perinatal WIN exposure induces a selective deficit in LTD in the PFC of adult offspring. $\boldsymbol{A}, \mathrm{A} 10-\mathrm{min}, 10-\mathrm{Hz}$ field stimulation of layer $2 / 3$ cells in the PFC of the adult offspring of sham-treated dams $(N=14)$ elicited a robust eCB-LTD at deep layer synapses. However, this same protocol failed to induce eCB-LTD in the adult offspring of dams treat with WIN $(N=14)$. $B$, fEPSP magnitude at baseline (-10-0 min) and LTD (35-40 min post-tetanus) values corresponding to the normalized values in $\boldsymbol{A}$ (two-way RM ANOVA, $F_{(1,24)}=16.58, p=0.0004$; Sidak's multiple comparisons test, $p=0.0332$ and $p=0.9412$, respectively). $\boldsymbol{C}$, LTD mediated by $\mathrm{mGlu}_{2 / 3}$ receptors (mGluR-LTD) is not altered in WIN-exposed offspring. mGluR-LTD, induced via a 10-min application of LY379268 (LY; 30 nM), produced a significant depression at deep layer synapses of the PFC in the adult offspring of both shamtreated and WIN-treated dams $(N=12,12$, respectively). $\boldsymbol{D}$, fEPSP magnitude at baseline ( $-10-0$ min) and LTD (30-40 min postdrug) values corresponding to the normalized values in $\boldsymbol{C}$. No differences were found between groups comparing the 10-min baseline period and the last $10 \mathrm{~min}$ of recording, however both groups exhibited a significant difference of fEPSP magnitude at baseline (i.e., $-10-0 \mathrm{~min}$ ) as compared with 30-40 min postdrug (two-way RM ANOVA, $F_{(1,11)}=96.69, p<0.0001$; Sidak's multiple comparisons test, $p<0.0001$ for both groups). $E$, A TBS protocol (five pulses at $100 \mathrm{hz}$, repeated four times) at layer $2 / 3 \mathrm{cells}$ in the PFC of the adult offspring of both sham-treated and WIN-treated dams elicited a robust LTP at deep layer synapses $(N=13,15$, respectively). $\boldsymbol{F}$, fEPSP magnitude at baseline $(-10-0 \mathrm{~min})$ and LTP $(30-40 \mathrm{~min}$ post-TBS) values corresponding to the normalized values in $\boldsymbol{E}$. Both groups exhibited significant differences between the fEPSP magnitude at 30-40 min as compared with - 10-0 min (twoway RM ANOVA, $F_{(1,25)}=1.737$; Sidak's multiple comparisons test, $p<0.0001$ for both groups); $* p<0.05$.

Table 3: PFC LTD data by sex

\begin{tabular}{llll}
\hline Condition & Test - measure (unit) & Value & $t$ test (M vs F) \\
\hline Sham male & eCB-LTD - normalized fEPSP (35-40 min post-tetanus) & $80.61 \pm 3.408 \mathrm{~N}=8$ & $p=0.5478$ \\
Sham female & eCB-LTD - normalized fEPSP (35-40 min post-tetanus) & $83.90 \pm 4.061 \mathrm{~N}=6$ & \\
WIN male & eCB-LTD - normalized fEPSP (35-40 min post-tetanus) & $103.9 \pm 8.384 \mathrm{~N}=6$ & $p=0.7042$ \\
WIN female & eCB-LTD - normalized fEPSP (35-40 min post-tetanus) & $108.8 \pm 9.488 \mathrm{~N}=7$ & \\
Sham male & mGlu2/3-LTD - normalized fEPSP (35-40 min postdrug) & $66.26 \pm 4.196 \mathrm{~N}=6$ & $p=0.8390$ \\
Sham female & mGlu2/3-LTD - normalized fEPSP (35-40 min postdrug) & $67.58 \pm 4.700 \mathrm{~N}=6$ & \\
WIN male & mGlu2/3-LTD - normalized fEPSP (35-40 min postdrug) & $67.48 \pm 4.156 \mathrm{~N}=7$ & $p=0.4467$ \\
WIN female & mGlu2/3-LTD - normalized fEPSP (35-40 min postdrug) & $72.00 \pm 3.900 \mathrm{~N}=5$ &
\end{tabular}


Table 4: PFC LTP data by sex

\begin{tabular}{llll}
\hline Condition & Test - measure (unit) & Value & $t$ test (M vs F) \\
\hline Sham male & TBS-LTP - normalized fEPSP (35-40 min post-tetanus) & $185.6 \pm 26.32 \mathrm{~N}=6$ & $p=0.3101$ \\
Sham female & TBS-LTP - normalized fEPSP (35-40 min post-tetanus) & $155.4 \pm 6.180 \mathrm{~N}=7$ & \\
WIN male & TBS-LTP - normalized fEPSP (35-40 min post-tetanus) & $168.0 \pm 11.98 \mathrm{~N}=9$ & $p=0.3917$ \\
WIN female & TBS-LTP - normalized fEPSP (35-40 min post-tetanus) & $152.6 \pm 12.58 \mathrm{~N}=6$ & \\
\hline
\end{tabular}

A

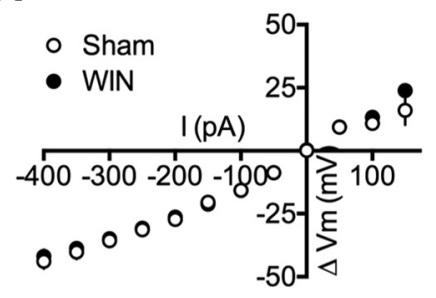

C

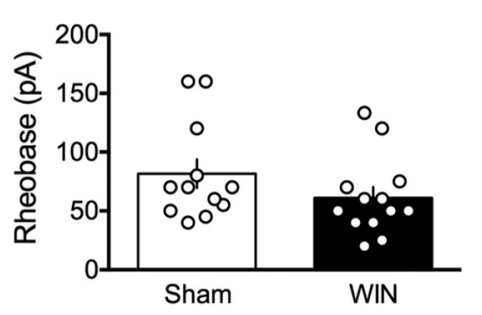

B

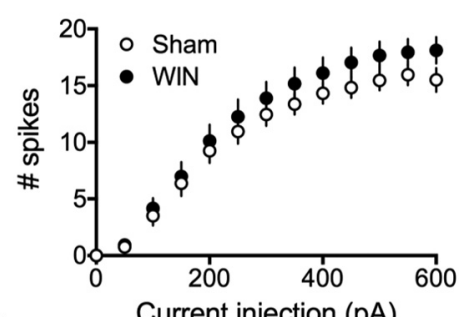

D

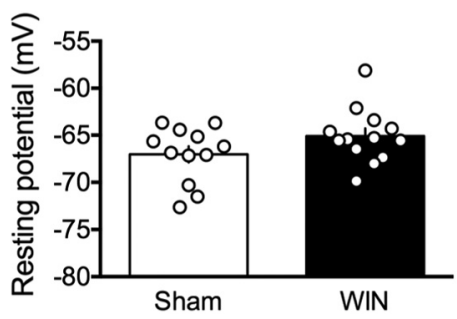

Figure 3. Perinatal WIN exposure does not alter properties of intrinsic excitability of deep layer pyramidal neurons in the PFC of adult offspring. $\boldsymbol{A}$, Current injection steps of $50 \mathrm{pA}$ from -400 to $150 \mathrm{pA}$ revealed no differences in the I-V relationship in pyramidal neurons of the PFC between the adult offspring of sham-treated and WIN-treated dams $(N=12,13$, respectively). $\boldsymbol{B}$, Action potentials elicited by progressive current injections from 0 to $600 \mathrm{pA}$ revealed no difference in the number of spikes elicited in pyramidal neurons of the PFC in slices obtained from the adult offspring of WIN-injected dams as compared with those from sham-treated dams $\left(N=13,12\right.$, respectively; two-way RM ANOVA, $\left.F_{(20,460)}=1.112, p=0.3328\right)$. $C$, Progressive current injections in 10-pA steps from 0 to $200 \mathrm{pA}$ revealed that the minimum current injection required to elicit an action potential (i.e., rheobase) did not differ in deep layer pyramidal neurons of PFC slices obtained from the adult offspring of WIN-treated, as compared with sham-treated, dams $(N=13,12$, respectively; two-tailed $t$ test, $p=0.1896)$. $D$, Similarly, no difference was found in the resting membrane potential of deep layer pyramidal cells in PFC slices obtained from the adult offspring of WIN-treated dams, as compared with those obtained from sham-treated dams $(N=13,12$, respectively; two-tailed $t$ test, $p=0.1123) ; * p<0.05$

\section{Perinatal exposure to WIN alters synaptic plasticity and cellular properties in the accumbens at adulthood}

Recent data have demonstrated that cannabinoids, experimenter-administered or self-administered, abolish LTD in the NAc (Mato et al., 2004, 2005; Neuhofer and Kalivas, 2018; Spencer et al., 2018). Thus, we sought to determine whether perinatal WIN exposure elicited similar deficits in LTD in the NAc at adulthood. We found that while the adult offspring of sham-treated dams exhibited robust LTD $30-40 \mathrm{~min}$ after a $10-\mathrm{min}, 10-\mathrm{Hz}$ stimulation, no such effect was found in the NAc of WIN-exposed rats (Fig. 4A,B; Table 6). Interestingly, unlike in the PFC, these alterations were accompanied by a significant reduction in the resting membrane potential of the principal neurons of the NAc, MSNs (Fig. 4F). No other parameters of cell excitability were found modified comparing MSNs in slices obtained from sham-exposed, as compared with WIN-exposed, rats (Fig. 4C-E; Table 7).

\section{Perinatal exposure to WIN enhances sucrose consumption at adulthood}

The NAc plays an important role in reward-associated behavior, and recent data indicate a relationship between LTD in the NAc and reward-seeking behavior including sucrose consumption (Bobadilla et al., 2017; Bilbao et al.,

Table 5: NAc LTD data by sex

\begin{tabular}{llll}
\hline Condition & Test - measure (unit) & Value & $t$ test (M vs F) \\
\hline Sham male & TBS-LTP - normalized fEPSP (35-40 min post-tetanus) & $82.37 \pm 6.937 \mathrm{~N}=5$ & $p=0.2893$ \\
Sham female & TBS-LTP - normalized fEPSP (35-40 min post-tetanus) & $71.51 \pm 6.589 \mathrm{~N}=5$ \\
WIN male & TBS-LTP - normalized fEPSP (35-40 min post-tetanus) & $110.9 \pm 6.257 \mathrm{~N}=5$ & $p=0.0714$ \\
WIN female & TBS-LTP - normalized fEPSP (35-40 min post-tetanus) & $95.53 \pm 3.039 \mathrm{~N}=4$
\end{tabular}


A

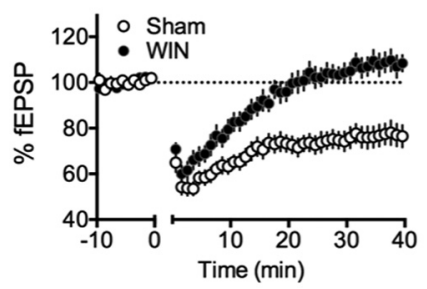

C

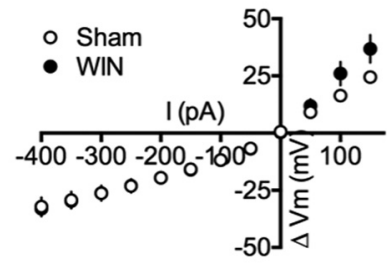

E

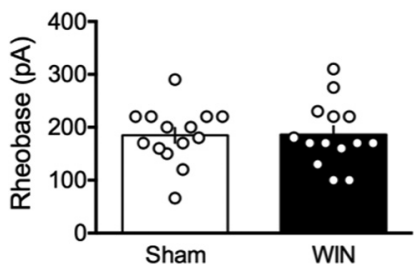

B

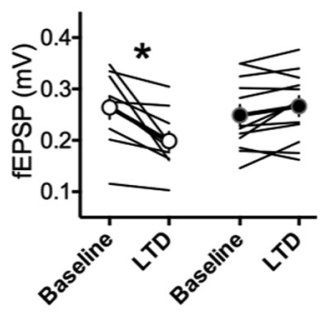

D

$\mathbf{F}$
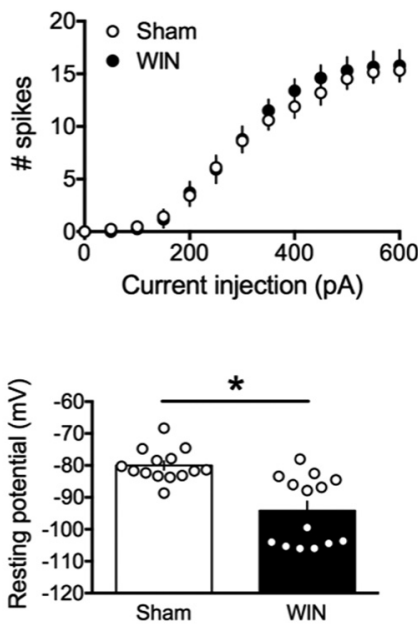

Figure 4. Perinatal WIN exposure abolishes LTD in the NAc of adult offspring and alters the resting membrane potential of NAc MSNs. A, A 10-min, $10-\mathrm{Hz}$ local field stimulation of the NAc of the adult offspring of sham-treated dams $(N=10)$ elicited a robust eCB-LTD. However, this same protocol failed to induce eCB-LTD in the adult offspring of dams treat with WIN $(N=12)$. $B, f E P S P$ magnitude at baseline (-10-0 min) and LTD (35-40 min post-tetanus) values corresponding to the normalized values in $\boldsymbol{A}$ (two-way RM ANOVA, $F_{(1,20)}=20.49, p=0.0002$; Sidak's multiple comparisons test, $p=0.0002$ and $p=0.3087$ for sham and WIN, respectively). $\boldsymbol{C}$, Current injection steps of $50 \mathrm{pA}$ from -400 to $150 \mathrm{pA}$ revealed no differences in the I-V relationship in MSNs of the NAc between the adult offspring of sham-treated and WIN-treated dams $(N=14,14$, respectively). $\boldsymbol{D}$, Action potentials elicited by progressive current injections from 0 to $600 \mathrm{pA}$ revealed no difference in the number of spikes elicited in pyramidal neurons of the PFC in slices obtained from the adult offspring of WIN-injected dams as compared with those from sham-treated dams $(N=14,14$, respectively; two-way RM ANOVA, $\left.F_{(20,250)}=0.6092, p=0.9071\right)$. $E$, Progressive current injections in 10 -pA steps from 0 to 200 pA revealed that the minimum current injection required to elicit an action potential (i.e., rheobase) did not differ in MSNs of NAc slices obtained from the adult offspring of WIN-treated, as compared with sham-treated, dams $(N=14,14$, respectively; two-tailed $t$ test, $p=0.9502)$. $\boldsymbol{F}$, However, MSNs in NAc slices obtained from the adult offspring of WIN-treated dams exhibited significantly lower resting membrane potentials than those obtained from sham-exposed offspring $(N=14$, 14, respectively; two-tailed $t$ test, $p=0.0003) ; * p<0.05$.

2020). Thus, we examined the magnitude of sucrose preference in a two-bottle choice paradigm in the adult offspring of sham-treated and WIN-treated dams. Here, we found that while both groups exhibited a preference for a $5 \%$ sucrose solution (as compared with plain water) and consumed similar total quantities of liquid during the test
(Fig. 5A; Table 8), the ratio of sucrose/water consumption was significantly higher in WIN- as compared with shamtreated adult offspring (Fig. 5B; Table 8). Thus, in addition to alterations to synaptic plasticity and intrinsic excitability of MSNs in the NAc, perinatal WIN exposure enhances reward-seeking behavior at adulthood.

Table 6: PFC intrinsic properties data by sex

\begin{tabular}{|c|c|c|c|}
\hline Condition & Test - measure (unit) & Value & $t$ test (M vs F) \\
\hline Sham male & Resting membrane potential (mV) & $-67.95 \pm 1.408 N=6$ & $p=0.3580$ \\
\hline Sham female & Resting membrane potential (mV) & $-66.11 \pm 0.9624 N=6$ & \\
\hline WIN male & Resting membrane potential (mV) & $-64.12 \pm 1.103 N=7$ & $p=0.2000$ \\
\hline WIN female & Resting membrane potential (mV) & $-66.22 \pm 1.077 N=6$ & \\
\hline Sham male & Rheobase $(\mathrm{pA})$ & $86,67 \pm 18.06 N=6$ & $p=0.7003$ \\
\hline Sham female & Rheobase (pA) & $76.67 \pm 17.64 N=6$ & \\
\hline WIN male & Rheobase (pA) & $66.19 \pm 16.34 N=7$ & $p=0.5487$ \\
\hline WIN female & Rheobase (pA) & $55.00 \pm 7.303 N=6$ & \\
\hline
\end{tabular}


Table 7: NAc intrinsic properties data by sex

\begin{tabular}{llll}
\hline Condition & Test - measure (unit) & Value & $t$ test (M vs F) \\
\hline Sham male & Resting membrane potential $(\mathrm{mV})$ & $-83.17 \pm 1.463 N=5$ & $p=0.0491$ \\
Sham female & Resting membrane potential $(\mathrm{mV})$ & $-78.26 \pm 1.684 N=9$ & \\
WIN male & Resting membrane potential $(\mathrm{mV})$ & $-93.95 \pm 3.909 N=8$ & $p=0.5281$ \\
WIN female & Resting membrane potential $(\mathrm{mV})$ & $-1004 \pm 8.945 N=6$ & \\
Sham male & Rheobase $(\mathrm{pA})$ & $199.2 \pm 36.64 N=5$ & $p=0.5827$ \\
Sham female & Rheobase $(\mathrm{pA})$ & $176.7 \pm 10.93 N=9$ & \\
WIN male & Rheobase $(\mathrm{pA})$ & $190.6 \pm 20.19 \mathrm{~N}=8$ & \\
WIN female & Rheobase $(\mathrm{pA})$ & $180.0 \pm 28.28 N=6$ & \\
\end{tabular}

A

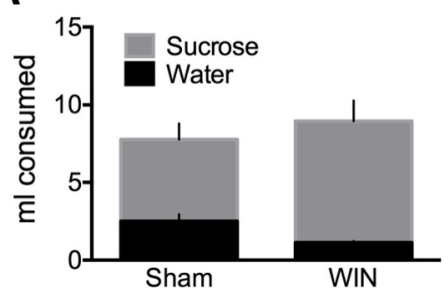

B

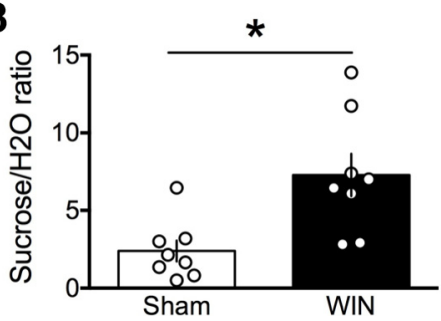

Figure 5. Perinatal WIN exposure increases sucrose preference in adult offspring. A, Total quantities of water and $5 \%$ sucrose solution $(\mathrm{ml})$ did not differ between the adult offspring of sham-treated or WIN-treated dams during a 20-min sucrose preference test $(N=8,8$, respectively). B. Preference for the $5 \%$ sucrose solution over water was significantly higher in WIN- as compared with sham-treated rats (two-tailed $t$ test, $p=0.0091$ ). ${ }^{\star} p<0.05$

\section{Discussion}

Here, using the synthetic cannabinoid WIN, we found that exposure to plant-derived phytocannabinoid THC via lactation induces behavioral and electrophysiological alterations lasting into adulthood. Specifically, we found altered social behavior, memory, and eCB-mediated synaptic plasticity in the PFC of adult offspring of dams administered WIN during the first $10 \mathrm{~d}$ of postnatal life. We also showing synaptic deficits and cellular alterations in the NAc along with enhanced sucrose preference, indicative of heightened reward seeking in WIN-exposed adults.

First, our behavioral analyses revealed that perinatal WIN exposure augments social preference in the adult offspring of WIN-treated dams. This result confirms the social augmentation seen following perinatal THC exposure (Scheyer et al., 2020a) but diverge from the effects of in utero THC exposure (i.e., social exploration was reduced). Such discrepancies point to potential differences in the sensitivity of developmental windows through the prenatal and early postnatal periods.

We also report that WIN exposure does not affect social memory, WIN abolishes novel object recognition at adulthood. Interestingly, social approach and memory is a complex behavior collating activity from diverse brain regions governing motivation and reward such as the amygdala (Adolphs, 2001) and NAc (Dölen et al., 2013). Indeed, augmentations in social approach behavior are often associated with decreased amygdalar function and signaling in the NAc, where oxytocin-mediated transmission is a key regulator of social approach and reward (Dölen et al., 2013) and is itself governed by the endocannabinoid system (ECS) (Wei et al., 2015). Previous data have highlighted the role of CCK interneuron dysfunction in WIN-mediated disruptions to social interaction (Vargish et al., 2017), a possible contributor to aberrant social behavior seen here that requires further investigation. Thus, variable

Table 8: Sucrose preference data by sex

\begin{tabular}{|c|c|c|c|}
\hline Condition & Test - measure (unit) & Value & $t$ test (M vs F) \\
\hline Sham male & Water consumed (ml) & $1.425 \pm 0.1181 N=4$ & $p=0.0045$ \\
\hline WIN male & Water consumed (ml) & $1.075 \pm 0.2287 N=4$ & $p=0.6953$ \\
\hline Sham male & Sucrose consumed (ml) & $3.550 \pm 1.533 N=4$ & $p=0.1069$ \\
\hline Sham female & Sucrose consumed (ml) & $7.000 \pm 0.7153 N=4$ & \\
\hline WIN male & Sucrose consumed (ml) & $8.800 \pm 1.564 N=4$ & $p=0.5118$ \\
\hline Sham female & Sucrose preference (ratio) & $2.039 \pm 0.3597 N=4$ & \\
\hline WIN male & Sucrose preference (ratio) & $8.674 \pm 1.744 N=4$ & $p=0.3462$ \\
\hline WIN female & Sucrose preference (ratio) & $5.885 \pm 2.089 \mathrm{~N}=4$ & \\
\hline
\end{tabular}


impacts on memory and exploration behavior are likely attributable to underlying differences in the driving circuitry.

Results from the current study examining the long-term consequences of perinatal WIN exposure adds to a preliminary report of dysfunctional eCB-LTD in the PFC of perinatally THC-exposed offspring (Scheyer et al., 2020a). In contrast with THC treatment however, perinatal WIN did not lead to an enhanced magnitude of mGlu2/3-LTD nor a loss of TBS-LTP in the WIN-exposed progeny at adulthood. Differences in the pharmacokinetics, bioavailability, and pharmacological profiles of WIN and THC may explain these differences (Pertwee, 2005). Indeed, while WIN is a highly selective agonist of CB1, THC exhibits a diverse range of activity from partial agonist targeting of CB1 and CB2 to activation of several transient receptor potential channels, orphan receptors, and the nuclear PPARy. Despite these subtle differences, these data and those from previous studies suggest that alteration of PFC synaptic plasticity and social behavior at adulthood are common endophenotypes of perinatal cannabinoid exposure (Hoffman et al., 2003; Vargish et al., 2017; Bara et al., 2018; Scheyer et al., 2020a,b).

Perinatal THC exposure decreases excitability of principle neurons of the PFC (Scheyer et al., 2020a) in a fashion similar to chronic adolescent THC exposure in mice (Pickel et al., 2020). Here, we found that no such differences followed perinatal WIN exposure. These data point to a dissociation between measures of intrinsic excitability and synaptic plasticity within the PFC, as changes in these domains appear independent. Thus, alternative explanations for the loss of eCB-LTD must be considered in light of a lack of changes to cell excitability, including alterations to receptor function or other changes to the ECS such as alterations in eCB tone.

The NAc is essential to reward-associated behavior and eCB-mediated LTD in the NAc core controls reward-seeking behavior (Bilbao et al., 2020). Here, we found that this eCB-LTD is ablated in the NAc of the adult offspring of WIN-treated dams. This finding is in line with multiple reports of altered LTD in the NAC of cannabinoid-exposed animals (Mato et al., 2004, 2005; Neuhofer and Kalivas, 2018; Spencer et al., 2018). In contrast with our recordings in PFC principal neurons, we observed a significant reduction in the resting membrane potential of NAC MSNs. Further, in examining the reward-seeking behavior of these WIN-exposed offspring, we also found that the ratio of sucrose/water consumption in the two-bottle choice task was significantly higher in WIN-treated, as compared with sham-treated, adult offspring. Thus, in the NAc of WIN-exposed progeny, the loss of eCB-LTD and associated cellexcitability modifications were paralleled by modifications of reward-seeking behavior at adulthood.

In conclusion, these results indicate that perinatal exposure via lactation to a synthetic cannabinoid reproduces some of the long-lasting deficits induced at multiple scales by THC. Augmented social behavior and a loss of eCB-LTD in the PFC are therefore similar consequences of perinatal exposure to both naturally occurring phytocannabinoids and synthetic cannabimimetics. Additionally, we found that WIN exposure ablates eCB-LTD in the NAc, where the resting membrane potential of MSNs was found to be significantly decreased. These findings may indeed correlate with an enhanced sucrose-preference among WIN-exposed offspring. Together, these findings further illustrate the vulnerability of the developing brain and, consequently, behavior, to earlylife insults to the endocannabinoid system via exposure to cannabinoid agonists.

\section{References}

Adolphs R (2001) The neurobiology of social cognition. Curr Opin Neurobiol 11:231-239.

Araque A, Castillo PE, Manzoni OJ, Tonini R (2017) Synaptic functions of endocannabinoid signaling in health and disease. Neuropharmacology 124:13-24.

Bara A, Manduca A, Bernabeu A, Borsoi M, Serviado M, Lassalle O, Murphy M, Wager-Miller J, Mackie K, Pelissier-Alicot A-L, Trezza V, Manzoni OJ (2018) Sex-dependent effects of in utero cannabinoid exposure on cortical function. Elife 7:e36234.

Bessa JM, Mesquita AR, Oliveira M, Pêgo JM, Cerqueira JJ, Palha JA, Almeida OFX, Sousa N (2009) A trans-dimensional approach to the behavioral aspects of depression. Front Behav Neurosci 3:1.

Bilbao A, Neuhofer D, Sepers M, Wei SP, Eisenhardt M, Hertle S, Lassalle O, Ramos-Uriarte A, Puente N, Lerner R, Thomazeau A, Grandes P, Lutz B, Manzoni OJ, Spanagel R (2020) Endocannabinoid LTD in accumbal D1 neurons mediates reward-seeking behavior. iScience 23:100951.

Bobadilla A-C, Garcia-Keller C, Heinsbroek JA, Scofield MD, Chareunsouk V, Monforton C, Kalivas PW (2017) Accumbens mechanisms for cued sucrose seeking. Neuropsychopharmacology 42:2377-2386.

Borsoi M, Manduca A, Bara A, Lassalle O, Pelissier-Alicot A-L, Manzoni OJ (2019) Sex differences in the behavioral and synaptic consequences of a single In vivo exposure to the synthetic cannabimimetic win55,212-2 at puberty and adulthood. Front Behav Neurosci 13:23.

Dölen G, Darvishzadeh A, Huang KW, Malenka RC (2013) Social reward requires coordinated activity of nucleus accumbens oxytocin and serotonin. Nature 501:179-184.

Goldman-Rakic PS (1991) Cellular and circuit basis of working memory in prefrontal cortex of nonhuman primates. Prog Brain Res 85:325-336.

Hoffman AF, Oz M, Caulder T, Lupica CR (2003) Functional tolerance and blockade of long-term depression at synapses in the nucleus accumbens after chronic cannabinoid exposure. J Neurosci 23:4815-4820.

Huang CC, Yang PC, Lin HJ, Hsu KS (2007) Repeated cocaine administration impairs group II metabotropic glutamate receptormediated long-term depression in rat medial prefrontal cortex. $J$ Neurosci 27:2958-2968.

Hurd YL, Manzoni OJ, Pletnikov MV, Lee FS, Bhattacharyya S, Melis M (2019) Cannabis and the developing brain: insights into its longlasting effects. J Neurosci 39:8250-8258.

Kasanetz F, Lafourcade M, Deroche-Gamonet V, Revest J-M, Berson N, Balado E, Fiancette J-F, Renault P, Piazza P-V, Manzoni OJ (2013) Prefrontal synaptic markers of cocaine addiction-like behavior in rats. Mol Psychiatry 18:729-737.

Martin HGS, Bernabeu A, Lassalle O, Bouille C, Beurrier C, PelissierAlicot AL, Manzoni OJ (2015) Endocannabinoids mediate muscarinic acetylcholine receptor-dependent long-term depression in the adult medial prefrontal cortex. Front Cell Neurosci 9:457-411.

Mato S, Chevaleyre V, Robbe D, Pazos A, Castillo PE, Manzoni OJ (2004) A single in-vivo exposure to $\Delta^{9} \mathrm{THC}$ blocks endocannabinoid-mediated synaptic plasticity. Nat Neurosci 7:585-586.

Mato S, Robbe D, Puente N, Grandes P, Manzoni OJ (2005) Presynaptic homeostatic plasticity rescues long-term depression 
after chronic $\Delta^{9}$-tetrahydrocannabinol exposure. J Neurosci 25:11619-11627.

Monleon S, D'Aquila P, Parra A, Simon VM, Brain PF, Willner P (1995) Attenuation of sucrose consumption in mice by chronic mild stress and its restoration by imipramine. Psychopharmacology (Berl) 117:453-457.

Neuhofer D, Kalivas P (2018) Metaplasticity at the addicted tetrapartite synapse: a common denominator of drug induced adaptations and potential treatment target for addiction. Neurobiol Learn Mem 154:97-111.

Pertwee RG (2005) Pharmacological actions of cannabinoids. Handb Exp Pharmacol 168:1-51.

Pickel VM, Bourie F, Chan J, Mackie K, Lane DA, Wang G (2020) Chronic adolescent exposure to $\Delta 9$-tetrahydrocannabinol decreases NMDA current and extrasynaptic plasmalemmal density of NMDA GluN1 subunits in the prelimbic cortex of adult male mice. Neuropsychopharmacology 45:374-383.

Renard J, Szkudlarek HJ, Kramar CP, Jobson CEL, Moura K, Rushlow WJ, Laviolette SR (2017) Adolescent THC exposure causes enduring prefrontal cortical disruption of GABAergic inhibition and dysregulation of sub-cortical dopamine function. Sci Rep 7:11420.

Scheyer AF, Melis M, Trezza V, Manzoni OJJ (2019) Consequences of perinatal cannabis exposure. Trends Neurosci 42:871-884.
Scheyer AF, Borsoi M, Pelissier- Alicot AL, Manzoni OJJ (2020a) Perinatal THC exposure via lactation induces lasting alterations to social behavior and prefrontal cortex function in rats at adulthood. Neuropsychopharmacology. Advance online publication. Retrieved May 19, 2020. doi: 10.1038/s41386-020-0716-x.

Scheyer AF, Borsoi M, Wager-Miller J, Pelissier-Alicot AL, Murphy MN, Mackie K, Manzoni OJJ (2020b) Cannabinoid exposure via lactation in rats disrupts perinatal programming of the gammaaminobutyric acid trajectory and select early-life behaviors. Biol Psychiatry 87:666-677.

Spencer S, Neuhofer D, Chioma VC, Garcia-Keller C, Schwartz DJ, Allen N, Scofield MD, Ortiz-Ithier T, Kalivas PW (2018) A model of $\Delta$ 9-tetrahydrocannabinol self-administration and reinstatement that alters synaptic plasticity in nucleus accumbens. Biol Psychiatry 84:601-610.

Vargish GA, Pelkey KA, Yuan X, Chittajallu R, Collins D, Fang C, McBain CJ (2017) Persistent inhibitory circuit defects and disrupted social behaviour following in utero exogenous cannabinoid exposure. Mol Psychiatry 22:56-67.

Wei D, Lee D, Cox CD, Karsten CA, Peñagarikano O, Geschwind DH, Gall CM, Piomelli D (2015) Endocannabinoid signaling mediates oxytocin-driven social reward. Proc Natl Acad Sci USA 112:14084-14089. 